\title{
Dynamics of Random Boolean Networks
}

\author{
James F. Lynch \\ Department of Mathematics and Computer Science \\ Box 5815 \\ Clarkson University \\ Potsdam, NY 13699-5815 \\ Telephone: 315-268-2374 \\ Fax: 315-268-2371 \\ email: jlynch@clarkson.edu
}

\begin{abstract}
Boolean networks are models of genetic regulatory networks. S. Kauffman based many of his claims about spontaneous self-organization in complex systems on simulations of randomly constructed Boolean networks. Some of these claims are precise mathematical statements. We analyze these statements using combinatorial methods and show that there is partial agreement with some of Kauffman's conclusions, but in other cases there is disagreement. Our key finding is an algebraic parameter that determines the likelihood of ordered behavior in a random Boolean network. There is a threshold such that when the parameter is less than the threshold, ordered behavior is prevalent, and when it is greater than the threshold, chaotic behavior is highly likely. When the parameter equals the threshold, some forms of ordered behavior persist, but others do not.
\end{abstract}

PACS codes: 87.10, 82.39.R, 02.10, 89.75.F

Keywords: Boolean networks, cellular metabolism, random graphs, stability

\section{Introduction}

The realization that the genome is a dynamic network, where some genes regulate the activity of other genes, is not new. It can be traced as far back as the Nobel prize winning discovery of the lac operon by Jacob and Monod [11]. Many other regulatory genes have been discovered since then, and a picture of the genome as a complex web of interacting genes has gradually emerged. Although the importance of this regulatory web was appreciated, mainstream molecular biology has largely focused on achieving a thorough understanding of the mechanisms underlying the interactions between individual molecular species. Until relatively recently, little attention was given to the study of the global behavior of molecular systems. This has changed with the recognition of systems biology as an important emerging discipline. 
Stuart Kauffman and René Thomas are among the first and most influential researchers to recognize the importance of the systems viewpoint in molecular biology. Much of their work pertains to Boolean networks. We will define Boolean networks more precisely later, but essentially they are highly simplified models of the genome, where each gene is modeled by a Boolean element called a gate. At any time, each gate is either active or inactive, i.e., its state is either 1 or 0 . The structure of the network determines how the activity of combinations of gates affects the activity of other gates. This is only an approximation of the behavior of actual genomic networks, but it was a reasonable starting point for several reasons. First, precise knowledge of the speed of molecular reactions was unavailable. Second, even if such knowledge had been available, the complexity of simulating reactions with many different rates would have overwhelmed the capabilities of existing computers. Both of these problems still exist, although much more is known now, and computing power has greatly increased in the last few decades.

A third reason for considering Boolean networks is that Kauffman and Thomas were interested in general properties of genomic networks and not in the detailed modeling of specific systems. Again, it makes sense to study the simplest and most basic model in order to find properties that apply to all gene nets.

Kauffman was interested in certain emergent properties of Boolean networks that are sometimes referred to as "spontaneous order" or "order for free." To summarize his thesis briefly, the genome is constructed from unreliable parts that are subject to damage from the environment. Further, their function can change as a result of mutation during reproduction. Yet the genome behaves in a robust and reliable manner. Kauffman argued that this order and stability was not solely the result of natural selection. There had to be a statistical tendency toward order and self-organization. In other words, natural selection acts on selforganizing systems rather than creating them. Without this tendency toward order, almost all mutations would be fatal, thus preventing evolution through natural selection. Kauffman has written extensively on this subject; his thinking is consolidated in his book [14].

Much of the evidence for Kauffman's thesis comes from computer simulations. The typical experiment consisted of randomly constructing a Boolean network subject to some constraints. Each gate's state was randomly initialized to 0 or 1 , and then the system ran synchronously. Since the network has a finite number of gates, each of which has two possible states, the network itself has a finite number of states, and it will eventually return to some state that it had been in previously. Since the network operates deterministically, it will keep returning to this state, repeating the same sequence of states indefinitely. This sequence of states is called the limit cycle.

According to Kauffman, the behavior of the network prior to entering its limit cycle is analogous to the behavior of an embryonic cell as it differentiates into its ultimate cell type, and the limit cycle is analogous to the differentiated cell's replication cycle.

Three measures of order were considered: 
1. The number of weak gates, i.e., gates that can be perturbed without changing the limit cycle that the network enters.

2. The number of gates that eventually freeze, i.e., they eventually stop changing state.

3. The size of the limit cycle.

Ordered behavior is characteristic of genomic and other biological networks. They have a large proportion of weak gates and frozen gates and a small limit cycle. Weak gates are a form of robustness - the ability to recover from small perturbations or errors. The presence of frozen gates indicates a degree of predictability in the network's behavior. A small limit cycle is another form of predictability: the system will repeat itself frequently. The opposite kinds of behavior are characteristic of non-biological, chaotic systems. A system where many of the gates are not weak shows sensitivity to initial conditions. The limit cycles in chaotic systems are very large, with many elements changing state unpredictably. Thus they are similar to strange attractors in continuous chaotic dynamical systems.

Since not all Boolean networks show ordered behavior, a basic problem is to find properties of networks that determine whether they will be ordered or chaotic. The degree of interconnection appears to be very important in this regard. This was varied in Kauffman's experiments by specifying the number of inputs each gate had, i.e., the number of gates whose states directly affected the gate. This number $k$ was fixed, and a random network was generated by choosing, for each gate, its $k$ input gates and assigning a Boolean function of $k$ arguments to the gate. ¿From the computer simulations, networks where $k \geq 3$ were chaotic, while those where $k \leq 2$ were very stable in the three senses described above. This is a kind of phase transition, where a change in microscopic conditions causes a change in macroscopic behavior.

Living cells seem to exhibit all three kinds of ordered behavior, and the typical gene is affected by only a few other genes. Thus the simulations provide evidence of spontaneous order in cells. In fact, Kauffman's computer simulations seemed to show a mathematical relationship between the number of gates and the size of the limit cycle in stable Boolean networks, which was paralleled in living cells by the number of genes and the duration of the replication cycle. The size of the limit cycle (or the duration of the replication cycle) appeared to be on the order of the square root of the number of gates (respectively genes).

One possible explanation for stability in Boolean networks where the gates had at most two inputs was that there are only 16 Boolean functions of two arguments, and two of those functions are constant, i.e., the function that always outputs 1 regardless of the values of its inputs, and the function that always outputs 0 . Thus a significant fraction $(1 / 8)$ of the gates in a random Boolean network with $k=2$ would be constant, and this could be the source of global stability of the network. Conversely, the chaotic behavior observed in nets with $k \geq 3$ could be a consequence of the much smaller proportion of constant gates. However, Kauffman also ran simulations of randomly constructed 
networks with $k=2$ but without constant gates, where the remaining 14 two argument functions were equally likely, and the results were similar to those where all 16 functions were used.

Kauffman proposed another category of functions as the source of order. He called these the canalyzing functions. A canalyzing function is a Boolean function for which there exists some argument and some Boolean value such that the output of the function is determined if the argument has that value. For example, the 2-argument OR function $x_{1} \vee x_{2}$ is canalyzing because if either argument has the value 1 , then the value of $x_{1} \vee x_{2}$ is 1 . Fourteen out of the sixteen 2-argument Boolean functions, including the constant functions, are canalyzing, but this proportion drops rapidly among Boolean functions with more than two arguments. Thus the hypothesis that nets with many canalyzing gates tend to be ordered, while those with few of them tend not to be, is consistent with the experimental results.

The sudden change in behavior between random Boolean networks with $k \leq 3$ and those with $k \leq 2$ is also evidence of the "edge of chaos" phenomenon that has been observed in many complex systems. The idea is that very stable and highly ordered systems are too simple and rigid to adapt to unpredictable influences from the environment; on the other hand, chaotic systems lack the robustness to maintain favorable states. Complex adaptive systems such as living cells are able to survive because they exist in a region between excessive stability and chaos. This theme has been elaborated on by Kauffman and many other researchers in complex systems, for example P. Bak [1] C. Langton [15], N. Packard [22], and S Wolfram [25].

All of these definitions and claims have precise mathematical formulations, so a natural question is whether the experimental results are supported by proofs. Indeed, this has been proven for the two extremes in the range of $k$. Networks with $k=1$ are highly ordered in the three senses above (S. Jaffe [12]). Networks with $k=n$, where $n$ is the number of gates, are equivalent to random functions on a set of size $2^{n}$. It can be shown that in this case, most gates will not be weak or frozen, and a classical result of B. Harris [9] implies that average limit cycle size is $(\sqrt{\pi / 8}) 2^{n / 2}$.

Interestingly, at about the same time that Kauffman started investigating random Boolean networks, the mathematical techniques for dealing with random networks were being developed by P. Erdős and A. Rényi [6, 7] and E. Gilbert [8], but it was about 30 years before any of these techniques were applied to the analysis of random Boolean networks. The first proofs of any of Kauffman's claims for networks with $1<k<n$ appear in an article co-authored by the mathematical biologist J. Cohen and the random graph theorist T. Euczak $[4]$.

Random graph theory is now a flourishing branch of combinatorics. The most extensively studied version of random graph is the independent edge model. In this version, there is a probability $p$ (which may depend on the number of vertices in the graph) such that for each pair of vertices independently, there is an undirected edge between them with probability $p$. Graph theorists have discovered many deep and interesting results about this kind of random graph, but 
it does not seem to be a good model of the random networks studied in biology, communications, and engineering. A major distinction is that the degree distribution of this kind of graph is Poisson, but the degree distributions of many real-world networks obey a power law. A better model for these situations may be random graphs with a specified degree distribution, which are considered in recent articles by M. Molloy and B. Reed [19, 20]. Some other shortcomings of the standard version of random graph pointed out by M. Newman, S. Strogatz, and D. Watts [21] are that it is undirected and has only one type of vertex. They develop some techniques for dealing with random directed graphs with vertices of several types. However, even this model lacks the structure needed to model the dynamic behavior of networks.

Kauffman's Boolean networks are a further extension of the models in [21] that do include this additional structure. The gates of a Boolean network are vertices assigned a type corresponding to a Boolean function, and the directed edges indicate the inputs to each gate. But instead of simply regarding each vertex as a static entity, we are interested in how the functions of the gates change the state of the network over time. Our random Boolean networks include Kauffman's networks as a special case. They are specified by a sequence of probabilities $p_{1}, p_{2}, \ldots$ whose sum is 1 , where for each gate independently, $p_{i}$ is the probability that it is assigned the $i$ th Boolean function. (We are assuming some canonical ordering of the finite Boolean functions.) Once each gate has been assigned its function, its indegree is determined by the number of arguments of the function, and its input gates are chosen at random using the uniform distribution. Lastly, a random initial state is chosen.

Our main result is an algebraic parameter, derived from the distribution $p_{1}, p_{2}, \ldots$, whose value determines the global behavior of the network. When the parameter is less than or equal to a certain threshold, ordered behavior of the first two kinds mentioned above is highly likely: almost all gates freeze quickly, and almost all gates can be perturbed without affecting the long-term behavior of the network. Conversely, if the parameter is larger than the threshold, the networks do not behave in such an ordered fashion. Our condition for stability actually implies forms of ordered behavior stronger than Kauffman's. That is, the gates freeze in time on the order of $\log n$, where $n$ is the number of gates, and the effect of a perturbation dies out within order $\log n$ steps. Consequently, the failure of our condition implies forms of disordered behavior that are weaker than the negations of Kauffman's.

We then apply our main results to the two classes of 2-input Boolean networks mentioned above. Here, our analysis verifies Kauffman's claims for networks that use all 16 of the 2-argument Boolean functions, but it casts doubt on similar claims for networks that use only the 14 nonconstant functions and the importance of canalyzing functions as a source of order.

Our techniques can also be applied to the limit cycle size. Here the picture is not as complete. However, it does provide the only case of an analytic result which definitely contradicts the experimental conclusions. Using the same parameter as above, when it is strictly less than the threshold, limit cycles are small. But when the parameter equals the threshold, limit cycles suddenly get 
very large. The analysis is quite involved and the details will be presented in another article. We sketch these results at the end of this article. We do not know the behavior of limit cycle sizes when the parameter is greater than the threshold.

Slightly weaker versions of the results in this article were presented in [17] and [18]. The main contribution of this article is a more general and uniform treatment of these results.

\section{Definitions}

A Boolean network $B$ is a 3 -tuple $\langle V, E, \mathbf{f}\rangle$ where $V$ is a set $\{1, \ldots, n\}$ for some natural number $n, E$ is a set of labeled directed edges on $V$, and $\mathbf{f}=\left(f_{1}, \ldots, f_{n}\right)$ is a sequence of Boolean functions such that for each $v \in V$, the number of arguments of $f_{v}$ is indeg $(v)$, the indegree of $v$ in $E$, i.e., the number of edges entering $v$. These edges are labeled $1, \ldots, \operatorname{indeg}(v)$. The interpretation is that $V$ is a collection of Boolean gates, $E$ describes their interconnections, and $\mathbf{f}$ describes their operation.

The gates update their states synchronously at discrete time steps $0,1, \ldots$ At any time $t$, each gate $v$ is in some state $x_{v} \in\{0,1\}$. Letting $\mathbf{x}=\left(x_{1}, \ldots, x_{n}\right)$, we say that $B$ is in state $\mathbf{x}$ at time $t$. Let $\operatorname{indeg}(v)=m$ and $u_{1}, u_{2}, \ldots, u_{m}$ be the gates such that for $i=1, \ldots, m,\left(u_{i}, v\right) \in E$ with label $i$. These are referred to as the in-gates of $v$. Then the state of $v$ at time $t+1$ is $y_{v}=f_{v}\left(x_{u_{1}}, \ldots, x_{u_{m}}\right)$. Letting $\mathbf{y}=\left(y_{1}, \ldots, y_{n}\right)$, we put $B(\mathbf{x})=\mathbf{y}$.

The following notation will be used to describe the dynamical properties of Boolean networks.

Definition 1. Let $\mathbf{x} \in\{0,1\}^{n}$, the set of sequences of $n$ 0's and 1's indexed by $1, \ldots, n$.

1. For $t=0,1, \ldots$, we put $B^{t}(\mathbf{x})$ for the state of $B$ at time $t$, given that its state at time 0 is $\mathbf{x}$. That is,

$$
\begin{aligned}
B^{0}(\mathbf{x}) & =\mathbf{x}, \text { and } \\
B^{t+1}(\mathbf{x}) & =B\left(B^{t}(\mathbf{x})\right) \text { for all } t .
\end{aligned}
$$

We also put $B_{v}^{t}(\mathbf{x})$ for $y_{v}$ where $\mathbf{y}=B^{t}(\mathbf{x})$.

2. Gate $v$ freezes to $y \in\{0,1\}$ in $t$ steps on input $\mathbf{x}$ if $B_{v}^{t^{\prime}}(\mathbf{x})=y$ for all $t^{\prime} \geq t$

3. For $\mathbf{x} \in\{0,1\}^{n}$ and $v \in\{1, \ldots, n\}$, we put $\mathbf{x}^{v}$ for the state which is identical to $\mathbf{x}$ except that $x_{v}^{v}=1-x_{v}$.

4. Let $u, v \in\{1, \ldots, n\}$ and $\mathbf{x} \in\{0,1\}^{n}$. We say that $v$ affects $u$ at time $t$ on input $\mathbf{x}$ if $B_{u}^{t}(\mathbf{x}) \neq B_{u}^{t}\left(\mathbf{x}^{v}\right)$. We put

$$
\begin{aligned}
& A_{+}^{t}(v, \mathbf{x})=\{u \in V: v \text { affects } u \text { at time } t \text { on input } \mathbf{x}\} \text { and } \\
& A_{-}^{t}(v, \mathbf{x})=\{u \in V: u \text { affects } v \text { at time } t \text { on input } \mathbf{x}\} .
\end{aligned}
$$


5. Gate $v$ is t-ineffective on input $\mathbf{x}$ if $A_{+}^{t}(v, \mathbf{x})=\emptyset$, i.e., $B^{t}(\mathbf{x})=B^{t}\left(\mathbf{x}^{v}\right)$.

Note that if $v$ is $t$-ineffective for some $t$, then it is weak. In analyzing the robustness of Boolean networks, we will estimate the number of $t$-ineffective gates, for suitable $t$, since this appears more tractable than estimating the number of weak gates. Similarly, instead of analyzing the frozen gates, we will consider a stronger property of gates.

We will be examining randomly constructed Boolean networks. The random model we use is sufficiently general to capture the particular classes of random Boolean networks in the literature. Let $\phi_{1}, \phi_{2}, \ldots$ be some ordering of all the finite Boolean functions, and let $p_{1}, p_{2}, \ldots$ be a sequence of probabilities such that $\sum_{i=1}^{\infty} p_{i}=1$. The selection of a random Boolean network with $n$ gates is a three stage process. First, each gate is independently assigned a Boolean function using the distribution $p_{1}, p_{2}, \ldots$ That is, for each $v=1, \ldots, n$ and $j=1,2, \ldots$, the probability that gate $v$ is assigned $\phi_{j}$ is $p_{j}$. The probabilities may depend on $n$, the number of gates in the network; that is, each probability is actually a function $p_{i}(n)$. For example, $p_{i}(n)=0$ for any $\phi_{i}$ with more than $n$ arguments. For simplicity of notation, we suppress the functional notation. Next, the in-gates for each gate are selected. If the gate has been assigned an $m$-argument function, then its in-gates are chosen from the $n(n-1) \ldots(n-m+$ 1) equally likely possibilities. Finally, random initial states are independently chosen for each gate.

We make several restrictions on the distribution $p_{1}, p_{2}, \ldots$ still consistent with the random networks in the literature. We assume that the average and variance of the number of arguments of a randomly selected Boolean function, or equivalently, the average and variance of the indegree of a gate, are finite. That is, letting each $\phi_{i}$ have $m_{i}$ arguments, $\sum_{i=1}^{\infty} p_{i} m_{i}^{2}<\infty$. Our methods require that the state of a random gate be independent of the time, i.e., for any gate $v$, the probability that $B_{v}^{t}(\mathbf{x})=1$ is a constant. This is ensured in the following way.

Definition 2. For any natural number $m$ and $\mathbf{x} \in\{0,1\}^{m}$, let $v(\mathbf{x})=\mid\{i: 1 \leq$ $i \leq m$ and $\left.x_{i}=1\right\} \mid$. Then for any $a \in[0,1]$,

$$
\rho(a)=\sum_{i=1}^{\infty} p_{i} \sum_{j=1}^{m_{i}} \sum_{\substack{\mathbf{x} \in\{0,1\}^{m_{i}} \\ \phi_{i}(\mathbf{x})=1}} a^{v(\mathbf{x})}(1-a)^{m_{i}-v(a)}
$$

is the probability that a random gate's state is 1 at time 1, given that each of its in-gates has probability a of being in state 1 at time 0.

Putting $\rho^{(t)}(a)=\rho(\rho(\ldots(a) \ldots))$ ( $\rho$ iterated $t$ times), we require that there exists $a \in[0,1]$ such that $\lim _{t \rightarrow \infty} \rho^{(t)}(b)=a$, where $b$ is the probability that a random gate's state is 1 at time 0 . This condition is easily seen to be satisfied by the random Boolean networks in the literature. They have $b=\frac{1}{2}$, and $a=\frac{1}{2}$ since every Boolean function and its negation have equal probability of being assigned to a gate. 
This class of random Boolean networks includes as special cases Kauffman's networks, networks with the classical random graph topology with edge probability $c n^{-a}, a \geq 1$ [2], networks with power law degree distribution $\propto d^{-c}$, $c>1$, smallworld networks $([23])$ and many of their variations $([21],[24]$,$) .$

If $\theta$ is a property of boolean nets then $\operatorname{pr}(\theta)$ denotes the probability that a random boolean network with $n$ gates satisfies $\theta$. If $\phi$ is also a property then $\operatorname{pr}(\theta \mid \phi)$ is the probability of $\theta$ over random boolean networks with $n$ gates, conditioned on $\phi$.

\section{Local Structure of Networks}

For small intervals of time, the dynamical properties of the network are determined by its "local" structure. Thus, the gates affected by a given gate over the time interval $0,1, \ldots, t$ lie in the portion of the network consisting of all gates reachable from the gate by a path in $E$ of length at most $t$. Similarly, the behavior of a gate over the same interval is determined by the portion consisting of all gates that can reach the gate by such a path. Of course, for large enough $t$, these portions will be the entire network. The next definitions capture these notions of locality.

Definition 3. 1. For any subset $I \subseteq V$,

$$
\begin{aligned}
S_{+}^{0}(I) & =I, \text { and } \\
S_{+}^{t+1}(I) & =\left\{u:(v, u) \in E \text { for some } v \in S_{+}^{t}(I)\right\} \text { for } t \geq 0 .
\end{aligned}
$$

That is, $S_{+}^{t}(I)$ is the set of gates at the ends of paths of length t that start in I. Similarly, $S_{-}^{t}(I)$ is the set of gates at the beginnings of paths of length $t$ that end in $I$.

2. Then

$$
\begin{aligned}
& N_{+}^{t}(I)=\bigcup_{s=0}^{t} S_{+}^{s}(I), \text { and } \\
& N_{-}^{t}(I)=\bigcup_{s=0}^{t} S_{-}^{s}(I)
\end{aligned}
$$

are the out- and in-neighborhoods respectively of I of radius $t$.

We put $S_{+}^{t}(v)$ for $S_{+}^{t}(\{v\})$ and similarly for the other notations. Thus the state of gate $v$ at time $t$ is determined by the states of the gates in $S_{-}^{t}(v)$ at time 0 and the functions assigned to the gates in $N_{-}^{t-1}(v)$.

We put $\log$ for $\log _{2}$. For the remainder of this article, $\alpha$ and $\beta$ will be positive constants satisfying $2 \alpha \log \delta+2 \beta<1$ and $\alpha \log \delta<\beta$, where $\delta=\mathbf{E}\left(m_{i}\right)$.

Lemma 1. Let $I \subseteq\{1, \ldots, n\},|I| \leq n^{\beta}$, and $t \leq \alpha \log n$. The following events have probability $1-o(1)$ : 
1. For every $v \in I, N_{+}^{t}(v)$ induces a tree in $\langle V, E\rangle$.

2. For every distinct $u, v \in I, N_{+}^{t}(u) \cap N_{+}^{t}(v)=\emptyset$.

3. For every $v \in I, N_{-}^{t}(v)$ induces a tree in $\langle V, E\rangle$.

4. For every distinct $u, v \in I, N_{-}^{t}(u) \cap N_{-}^{t}(v)=\emptyset$.

Proof. The lemma follows by showing that each of these events fails with probability $o(1)$. The calculations are similar for all events, and we show the work only for event 1 .

If 1 . fails, then there exist distinct gates $v_{1}, \ldots, v_{s}$ such that

$s \leq \alpha \log n$,

for $i=1, \ldots, s-1, v_{i}$ is an in-gate of $v_{i+1}$, and

$v_{1} \in I$,

and distinct gates $w_{1}, \ldots, w_{r}$ such that

$r \leq \alpha \log n$,

for $i=1, \ldots, r-1, w_{i}$ is an in-gate of $w_{i+1}$,

$w_{1} \in\left\{v_{1}, \ldots, v_{s}\right\}$,

$\left\{w_{2}, \ldots, w_{r}\right\} \cap\left\{v_{1}, \ldots, v_{s}\right\}=\emptyset$, and

there is a labelled edge $\left(w_{r}, v_{s}\right)$ distinct from the labelled edge $\left(v_{s-1}, v_{s}\right)$.

Now $s$ and $r$ can be chosen in $O\left((\log n)^{2}\right)$ ways. The gates $v_{1}, \ldots, v_{s}$ and $w_{1}, \ldots, w_{r}$ can be chosen in $O\left(n^{s+r-2+\beta} \log n\right)$ ways. For each $i=1, \ldots, s-2$, the probability that $v_{i}$ is an in-gate of $v_{i+1}$ is

$$
\sum_{i=1}^{\infty} p_{i} \frac{m_{i}}{n}=\frac{\delta}{n}
$$

Similarly, the probability that each $w_{i}$ is an in-gate of $w_{i+1}$ for $i=1, \ldots, r-1$ is $\delta / n$. The probability that both $v_{s-1}$ and $w_{r}$ are in-gates of $v_{s}$ is

$$
\sum_{i=1}^{\infty} p_{i} \frac{m_{i}\left(m_{i}-1\right)}{n(n-1)}=O\left(n^{-2}\right) .
$$

Altogether, the probability that 1 . fails is

$$
\begin{aligned}
O\left((\log n)^{3} \times n^{s+r-2+\beta} \times(\delta / n)^{s+r-3} \times n^{-2}\right) & =O\left((\log n)^{3} \delta^{2 \alpha \log n} n^{\beta-1}\right) \\
& =O\left((\log n)^{3} n^{2 \alpha \log \delta+\beta-1}\right) \\
& =o(1) .
\end{aligned}
$$




\section{Branching Processes}

As we showed, for $t$ not large compared to $n$, the typical $N_{+}^{t}(v)$ induces a tree in a Boolean network with $n$ gates. A perturbation of the state of such $v$ may cause perturbations to the states of $S_{+}^{1}(v)$ in the next step, then $S_{+}^{2}(v)$, and so on, in a "wave" that propagates through $N_{+}^{t}(v)$. It is possible that this wave dies out and the effects of the perturbation are transient, i.e., gate $v$ is $t$-ineffective. We will show that this behavior can be approximated by a branching process. Then, by applying basic results about branching processes, we will derive our results about ineffective gates. Similarly, $N_{-}^{t}(v)$ induces a tree for almost all $v$. In this case, the gates that affect $v$ at times $0, \ldots, t$ are the successive generations in a branching process that propagates backwards through $N_{-}^{t}(v)$.

We will summarize the results about branching processes that we need. For more information on branching processes, see T. Harris [10].

A branching process can be identified with a rooted labelled tree. The tree may have infinite branches. Each node will be labelled with the unique path from the root to that node. That is, the root is labelled with the null sequence. If the root has $k$ children, they are labelled with the sequences $(1),(2), \ldots,(k)$. If the second child of the root has $l$ children, then they are labelled with the sequences $(2,1),(2,2), \ldots,(2, l)$, and so on. Generation $t$ consists of all nodes labelled with a sequence of length $t$. The number of children of any node is independent of the number of children of any other node, but the probability of having a certain number of children is the same for all nodes. Thus the probability space of a branching process is determined by a sequence $\left(q_{k}: k=0,1, \ldots\right)$ where $q_{k}$ is the probability that a node has $k$ children. The probability measure on this space will be denoted by bpr. In describing events in this space, $P$ will denote a branching process. If $\chi$ is a property of branching processes, $P=\chi$ means $\chi$ holds for $P$, and $\operatorname{bpr}(P \models \chi)$ is the probability that $\chi$ holds.

For $t \geq 0, P \uparrow t$ will be the finite labelled tree which is $P$ restricted to its first $t$ generations. $Z_{t}$ will be the random variable which is the size of generation $t$, i.e., the number of nodes of depth $t$.

The generating function of the branching process is the series

$$
F(z)=\sum_{k=0}^{\infty} q_{k} z^{k} .
$$

That is, $F(z)$ is the probability generating function of $Z_{1}$ since $q_{k}=\operatorname{bpr}\left(Z_{1}=\right.$ $k)$. A basic result is that the $t$-th iterate of $F(z)$ is the probability generating function of $Z_{t}$. The iterates of $F(z)$ are defined by

$$
\begin{aligned}
F_{0}(z) & =z \text { and } \\
F_{t+1}(z) & =F\left(F_{t}(z)\right) \text { for } t \geq 0 .
\end{aligned}
$$

Then 
Theorem 1. The probability generating function of $Z_{t}$ is $F_{t}(z)$, i.e.,

$$
F_{t}(z)=\sum_{k=0}^{\infty} \operatorname{bpr}\left(Z_{t}=k\right) z^{k} .
$$

This enables us to express the moments of $Z_{t}$ in terms of the moments of $Z_{1}$, which in turn have simple representations in terms of the derivatives of $F(z)$. Let $\mu$ and $\sigma^{2}$ be the first and second moments of $Z_{1}$, that is, $\mu=\mathbf{E}\left(Z_{1}\right)$ and $\sigma^{2}=\operatorname{var}\left(Z_{1}\right)$.

Theorem 2. We have

$$
\begin{aligned}
\mu & =F^{\prime}(1) \text { and } \\
\sigma^{2} & =F^{\prime \prime}(1)+F^{\prime}(1)-\left(F^{\prime}(1)\right)^{2} .
\end{aligned}
$$

More generally, for all $t \geq 0$, the first and second moments of $Z_{t}$ are

$$
\begin{aligned}
\mathbf{E}\left(Z_{t}\right) & =\mu^{t} \text { and } \\
\operatorname{var}\left(Z_{t}\right) & = \begin{cases}\frac{\sigma^{2} \mu^{t}\left(\mu^{t}-1\right)}{\mu^{2}-\mu} & \text { if } \mu \neq 1, \\
t \sigma^{2} & \text { if } \mu=1 .\end{cases}
\end{aligned}
$$

Our analysis of frozen and ineffective gates uses two branching processes, both of which are defined in terms of a parameter that characterizes the tendency of gates to be influenced (regulated) by their in-gates.

Definition 4. Let $f\left(x_{1}, \ldots, x_{m}\right)$ be a Boolean function of $m$ arguments, and $\mathbf{x}=\left(x_{1}, \ldots, x_{m}\right) \in\{0,1\}^{m}$ be an assignment of 0's and 1's to its arguments. For $i \in\{1, \ldots, m\}$, we say that argument $i$ directly affects $f$ on input $\mathbf{x}$ if $f(\mathbf{x}) \neq f\left(\mathbf{x}^{i}\right)$. We put $\gamma(f, \mathbf{x})$ for the number of $i$ 's that directly affect $f$ on input $\mathbf{x}$. We extend this notion to gates in a Boolean network in the obvious way. Given a Boolean network $B$ where gate $v$ has in-gates $u_{1}, \ldots, u_{m}$ and state $\mathbf{x} \in\{0,1\}^{n}$, for $i=1, \ldots, m, u_{i}$ directly affects $v$ on input $\mathbf{x}$ if $B_{v}(\mathbf{x}) \neq$ $B_{v}\left(\mathbf{x}^{u_{i}}\right)$.

Let

$$
\lambda=\sum_{i=1}^{\infty} p_{i} \sum_{\mathbf{x} \in\{0,1\}^{m_{i}}} \gamma\left(\phi_{i}, \mathbf{x}\right) a^{v(\mathbf{x})}(1-a)^{m_{i}-v(a)} .
$$

Thus $\lambda$ may be regarded as the average number of arguments that directly affect a random Boolean function with a random input.

\section{$5 \quad$ Ineffective Gates}

As we showed, for sufficiently small $I$ and $t$, the "typical" $N_{+}^{t}(I)$ and $N_{-}^{t}(I)$ induce a forest on $\langle V, E\rangle$, i.e., there are no directed or undirected cycles among their gates. If this is the case for $N_{+}^{t}(v)$, then we can give a simple recursive definition of $A_{+}^{t}(v, \mathbf{x})$. 
Lemma 2. Assume $N_{+}^{t}(v)$ induces a tree on $E$. Then for any $s \leq t$, any $\mathbf{x} \in\{0,1\}^{n}$, and any gate $u \in S_{+}^{s}(v), v$ affects $u$ at time $s$ on input $\mathbf{x}$ if and only if

1. $s=0$ and $u=v$, or

2. $s>0$ and, letting $w$ be the unique gate such that $w \in S_{+}^{s-1}(v) \cap S_{-}^{1}(u)$, $v$ affects $w$ at time $s-1$ on input $\mathbf{x}$, and $w$ directly affects $u$ on input $B^{s-1}(\mathbf{x})$.

We will use the branching process defined as follows.

$$
q_{k}=\frac{\lambda^{k}}{k !} e^{-\lambda}
$$

for $k=0,1, \ldots$ Therefore $F(z)=e^{\lambda z-\lambda}$. ¿From Theorem 2,

$$
\begin{aligned}
\mu & =\lambda, \\
\sigma^{2} & =\lambda, \\
\mathbf{E}\left(Z_{t}\right) & =\lambda^{t}, \text { and } \\
\operatorname{var}\left(Z_{t}\right) & = \begin{cases}\frac{\lambda^{t}\left(\lambda^{t}-1\right)}{\lambda-1} & \text { if } \mu \neq 1, \\
t \lambda & \text { if } \mu=1 .\end{cases}
\end{aligned}
$$

Definition 5. Let $T$ be a labelled tree of height $t, B=\langle V, E, \mathbf{f}\rangle$ be a Boolean network, and $\mathbf{x} \in\{0,1\}^{n}$ be its state. For $v \in\{1, \ldots, n\}$, we put $T \Longrightarrow v$ if

$$
N_{-}^{t}\left(A^{t}(v, \mathbf{x})\right) \text { induces a tree in }\langle V, E\rangle \text {, and }
$$

there is an isomorphism from $T$ onto $\left\langle A_{+}^{t}(v, \mathbf{x}), E\right\rangle$.

Lemma 3. If $|T| \leq n^{\beta}$ and the height of $T$ is $t \leq \alpha \log n$, then for all $\mathbf{x} \in$ $\{0,1\}^{n}, \operatorname{pr}(T \Longrightarrow v)=\operatorname{bpr}(P \nmid t \cong T)(1+o(1))$.

Proof. By Lemma 1, if there is an isomorphism $\tau$ from $T$ onto $\left\langle A_{+}^{t}(v, \mathbf{x}), E\right\rangle$, then almost surely $N_{-}^{t}\left(A_{+}^{t}(v, \mathbf{x})\right)$ induces a tree in $\langle V, E\rangle$. Thus we need only analyze the probability that $\tau$ exists. Let $u_{1}, \ldots, u_{h}$ be the non-leaf nodes of $T$, in lexicographic order. The construction of $\tau$ is recursive and proceeds in stages $1, \ldots, h$. At each stage $s, \tau\left(u_{s}\right)$ has been defined at some previous stage, and it is extended to the children of $u_{s}$. (At stage $1, \tau\left(u_{1}\right)=v$ has already been defined.) Also, the Boolean functions assigned to these children are selected.

Thus, assume that at stage $s, \tau\left(u_{1}\right), \ldots, \tau\left(u_{K_{s}}\right)$ have already been defined, where $s \leq K_{s}$. Let $u_{s}$ have $k_{s}$ children. Then there are $\left(\begin{array}{c}n-K_{s} \\ k_{s}\end{array}\right)$ ways of selecting the children of $\tau\left(u_{s}\right)$ in $\langle V, E\rangle$. Having chosen these children, we next assign Boolean functions to them. Independently, for each child $w$ of $\tau\left(u_{s}\right)$, let $\phi_{i}$ be assigned to it. This event has probability $p_{i}$, and for $j=1, \ldots, m_{i}$, the probability that $\tau\left(u_{s}\right)$ is the $j$ th in-gate of $w$ is $1 / n$. Summing over all $i$, we get 
the probability that $\tau\left(u_{s}\right)$ directly affects $w$ :

$\sum_{i=1}^{\infty} p_{i} \sum_{\mathbf{x} \in\{0,1\}^{m_{i}}} \sum_{j=1}^{m_{i}} \operatorname{pr}\left(\tau\left(u_{s}\right)\right.$ is the $j$ th in-gate of $w$, the initial state is $\mathbf{x}$ and $u_{s}$ directly affects $w$ on input $\left.\mathbf{x} \mid f_{w}=\phi_{i}\right)$

$$
\begin{aligned}
& =\sum_{i=1}^{\infty} \frac{p_{i}}{n} \sum_{\mathbf{x} \in\{0,1\}^{m_{i}}} \gamma\left(\phi_{i}, \mathbf{x}\right) a^{v(\mathbf{x})}(1-a)^{m_{i}-v(a)} \\
& =\frac{\lambda}{n}
\end{aligned}
$$

Therefore the probability that these $k_{s}$ gates are directly affected by $\tau\left(u_{s}\right)$ is $(\lambda / n)^{k_{s}}$.

Since the events of assigning Boolean functions to all the gates are independent, the probability that the selected gates belong to $A_{+}^{t}(v, \mathbf{x})$ is

$$
\begin{aligned}
\prod_{s=1}^{h}\left(\begin{array}{c}
n-K_{s} \\
k_{s}
\end{array}\right)\left(\frac{\lambda}{n}\right)^{k_{s}} & =\left(\prod_{s=1}^{h} \frac{\lambda^{k_{s}}}{k_{s} !}\right)\left(1-\frac{O\left(n^{\beta}\right)}{n}\right)^{O\left(n^{\beta}\right)} \\
& =\left(\prod_{s=1}^{h} \frac{\lambda^{k_{s}}}{k_{s} !}\right)\left(1-O\left(n^{2 \beta-1}\right)\right) .
\end{aligned}
$$

The probability that no other gates are in $A_{+}^{t}(v, \mathbf{x})$ is

$$
\left(1-\frac{\lambda h}{n}\right)^{n-|T|}=e^{-\lambda h}\left(1+O\left(n^{2 \beta-1}\right)\right) .
$$

Therefore

$$
\begin{aligned}
\operatorname{pr}(T \Longrightarrow v) & =\left(\prod_{s=1}^{h} \frac{\lambda^{k_{s}}}{k_{s} !} e^{-\lambda}\right)(1+o(1)) \\
& =\operatorname{bpr}(P \uparrow t \cong T)(1+o(1))
\end{aligned}
$$

We say that a property $\chi$ of branching processes depends only on the first $t$ generations if, for any two branching processes $P_{1}$ and $P_{2}$ such that $P_{1} \uparrow t \cong$ $P_{2}\left\lceil t\right.$, either $P_{1} \models \chi$ and $P_{2} \models \chi$, or $P_{1} \not \models \chi$ and $P_{2} \not \models \chi$. Thus $\chi$ can be identified with a set of labelled trees of depth at most $t$. We will also use the notation $\left\langle A_{+}^{t}(v, \mathbf{x}), E\right\rangle \models \chi$ to mean $\left\langle A_{+}^{t}(v, \mathbf{x}), E\right\rangle$ induces a tree in $\langle V, E\rangle$ whose corresponding branching process satisfies $\chi$.

Theorem 3. Let $\chi$ be a property of branching processes that depends only on the first $\alpha \log n$ generations. Then for all $\mathbf{x} \in\{0,1\}^{n}$

$$
\operatorname{pr}\left(\left\langle A_{+}^{t}(v, \mathbf{x}), E\right\rangle \models \chi\right)=\operatorname{bpr}(P \models \chi)+o(1) .
$$


Proof. By the previous lemma, it suffices to show that $\operatorname{bpr}\left(|P| \alpha \log n \mid \geq n^{\beta}\right)=$ $o(1)$.

If $|P| \alpha \log n \mid \geq n^{\beta}$, then $Z_{t} \geq n^{\beta} /(\alpha \log n)$ for some $t=1, \ldots, \alpha \log n$. Since $\mathbf{E}\left(Z_{t}\right)=\lambda^{t} \leq \delta^{t} \leq n^{\alpha \log \delta} \ll n^{\beta} /(\alpha \log n)$,

$$
\begin{aligned}
\operatorname{pr}\left(Z_{t} \geq n^{\beta} /(\alpha \log n)\right) \leq & \frac{\operatorname{var}\left(Z_{t}\right)}{\left(n^{\beta} /(\alpha \log n)-\mathbf{E}\left(Z_{t}\right)\right)^{2}} \text { by Chebyshev's inequality } \\
& =\left\{\begin{array}{l}
\frac{\lambda^{2 t-1}+\lambda^{2 t-2}+\cdots+\lambda^{t}}{\left(n^{\beta} /(\alpha \log n)-\lambda^{t}\right)^{2}} \text { if } \lambda \neq 1 \\
\frac{t \lambda}{\left(n^{\beta} /(\alpha \log n)-\lambda^{t}\right)^{2}} \text { if } \lambda=1 \\
=o(1 / \log n) \text { in either case. }
\end{array}\right.
\end{aligned}
$$

A gate $v$ such that $N_{-}^{\alpha \log n}\left(A_{+}^{\alpha \log n}(v, \mathbf{x})\right)$ is acyclic is $\alpha \log n$-ineffective if and only if its corresponding branching process is extinct within $\alpha \log n$ generations. Clearly this depends only on the first $\alpha \log n$ generations, so Theorem 3 applies. By basic results from branching process theory, the probability of extinction in $t$ generations is $\operatorname{bpr}\left(Z_{t}=0\right)=F_{t}(0)$, and $\lim _{t \rightarrow \infty} F_{t}(0)=r$, where $r$ is the smallest nonnegative root of $z=F(z)$. Further, when $\mu \leq 1, r=1$, and when $\mu>1, r<1$. Therefore

Theorem 4. There is a constant $r$ such that for all $\mathbf{x} \in\{0,1\}^{n}$

$$
\lim _{n \rightarrow \infty} \operatorname{pr}(v \text { is } \alpha \log n \text {-ineffective })=r .
$$

When $\lambda \leq 1, r=1$, and when $\lambda>1, r<1$.

Corollary 1. The expected number of $\alpha \log n$-ineffective gates in a random Boolean network is asymptotic to $r n$.

A stronger result is

Corollary 2. The number of $\alpha \log n$-ineffective gates in almost all Boolean networks is asymptotic to rn.

That is, there is a function $\varepsilon(n)$ such that $\varepsilon(n) \rightarrow 0$ and, letting the random variable $X_{n}$ be the number of $\alpha \log n$-ineffective gates in a random Boolean network with $n$ gates,

$$
\lim _{n \rightarrow \infty} \operatorname{pr}\left(\left|X_{n}-r n\right| \leq n \varepsilon(n)\right)=1 .
$$

Proof. By the previous corollary,

$$
\mathbf{E}\left(X_{n}\right)=r n+n \varepsilon(n),
$$


where $\varepsilon(n)$ is a function such that $\lim _{n \rightarrow \infty} \varepsilon(n)=0$. When $\lambda \leq 1, r=1$, so, letting the random variable $Y_{n}=n-X_{n}$, by Markov's inequality

$$
\operatorname{pr}\left(Y_{n} \geq n \sqrt{|\varepsilon(n)|}\right)=O(\sqrt{|\varepsilon(n)|}) .
$$

Therefore the corollary holds for $\lambda \leq 1$.

When $\lambda>1, r<1$, and we need to estimate $\operatorname{var}\left(X_{n}\right)$. Using methods similar to those in the proofs of Lemma 1 and Theorems 3 and 4 it can be shown that, for any two distinct gates $u$ and $v$, almost surely $N_{-}^{\alpha \log n}\left(A_{+}^{\alpha \log n}(u, \mathbf{x})\right)$ and $N_{-}^{\alpha \log n}\left(A_{+}^{\alpha \log n}(v, \mathbf{x})\right)$ are acyclic, their intersection is empty, and

$$
\lim _{n \rightarrow \infty} \operatorname{pr}(u \text { and } v \text { are } \alpha \log n \text {-weak })=r^{2} .
$$

Therefore

$$
\operatorname{var}\left(X_{n}\right)=r(1-r) n+n^{2} \varepsilon^{\prime}(n)
$$

for some function $\varepsilon^{\prime}(n) \rightarrow 0$. By Chebyshev's inequality

$$
\begin{aligned}
\operatorname{pr}\left(\left|X_{n}-r n-n \varepsilon(n)\right|>n \sqrt[4]{\left|\varepsilon^{\prime}(n)\right|}\right) & \leq \frac{r(1-r) n+n^{2} \varepsilon^{\prime}(n)}{n^{2} \sqrt{\left|\varepsilon^{\prime}(n)\right|}} \\
& \rightarrow 0,
\end{aligned}
$$

and the corollary also holds for $\lambda>1$.

When $\lambda>1$, it is also true that most of the $\alpha \log n$-effective gates affect many other gates when perturbed.

Corollary 3. Let $\lambda>1$. For almost all random Boolean networks, if gate $v$ is $\alpha \log n$-effective, then there is a positive $W$ such that for $t \leq \alpha \log n$, the number of gates affected by $v$ at time $t$ is asymptotic to $W \lambda^{t}$.

Proof. For $t \geq 0$, let $W_{t}=Z_{t} / \mu^{t}$ (= $Z_{t} / \lambda^{t}$ in our case). Again by basic results from branching process theory, there is a random variable $W$ such that

$$
\begin{aligned}
\operatorname{bpr}\left(\lim _{t \rightarrow \infty} W_{t}=W\right) & =1 \text { and } \\
\lim _{t \rightarrow \infty} \operatorname{bpr}\left(Z_{t} \neq 0 \text { and } W=0\right) & =0 .
\end{aligned}
$$

¿From this the corollary follows.

\section{Frozen Gates}

Estimating the number of gates that freeze seems to be quite difficult. However, there is a condition on gates whose in-neighborhoods are trees that implies freezing and which is amenable to combinatorial analysis.

For the remainder of this section, $t$ will represent a natural number in the range $0, \ldots, \alpha \log n$, and $y$ will be a variable taking on the values 0 and 1 . Given a Boolean function $\phi\left(x_{1}, \ldots, x_{m}\right)$ and $\mathbf{x}=\left(x_{1}, \ldots, x_{m}\right) \in\{0,1, *\}^{m}$, we 
say that $\mathbf{x}$ forces $\phi$ to $y$ if, for all $\mathbf{x}^{\prime} \in\{0,1\}^{m}$ such that $x_{i}=x_{i}^{\prime}$ whenever $x_{i} \neq *, \phi\left(\mathbf{x}^{\prime}\right)=y$. The $*$ 's are "don't care" values, meaning their value does not affect the value of $\phi$ whenever the remaining arguments agree with $\mathbf{x}$. For example, $\phi$ is forced by every $\mathbf{x} \in\{0,1\}^{m}$; if $\phi$ is a constant function, then it is forced by every $\mathbf{x} \in\{0,1, *\}^{m}$; if $\phi\left(x_{1}, x_{2}\right)=x_{1} \vee x_{2}$, then it is forced to 0 by $(0,0)$ and to 1 by $(0,1),(1,0),(1,1),(1, *)$, and $(*, 1)$. We can now give a recursive definition of forcing for the gates of a Boolean network.

Definition 6. A gate $v$ is forced to $y$ in 0 steps if $f_{v}$ is the constant function $y$.

For $t \geq 0, v$ is forced to $y$ in $t+1$ steps if, letting $u_{1}, \ldots, u_{m}$ be its in-gates, there is $\mathbf{x} \in\{0,1, *\}^{m}$ such that $\mathbf{x}$ forces $f_{v}$ to $y$ and for each $i=1, \ldots, m$ such that $x_{i} \neq *, f_{u_{i}}$ is forced to $x_{i}$ in $t$ steps. We say that $v$ is forced (in some number of steps) if it is forced to 0 or 1.

It is clear that forcing is a stronger condition than freezing.

Lemma 4. If a gate in a Boolean network is forced to $y$ in $t$ steps, then it freezes to $y$ in $t$ steps.

When the in-neighborhood of a gate is a tree, forcing is related to the branching process where the children of a gate are its in-gates that directly affect it. The next lemma states that forcing is equivalent to extinction in this branching process. We put $A_{-}^{t}(v)=\bigcup_{\mathbf{x}} A_{-}^{t}(v, \mathbf{x})$.

Lemma 5. If $N_{-}^{t}(v)$ induces a tree, then $v$ is forced in $t$ steps if and only if $A_{-}^{t}(v)=\emptyset$.

Proof. The "only if" direction of the proof is immediate from the definitions.

To prove the other direction, we use induction on $t$. When $t=0$, it is again immediate from the definitions.

Now assume the result holds for $t, N_{-}^{t+1}(v)$ induces a tree, and $v$ is not forced in $t+1$ steps. Let $u_{1}, \ldots, u_{m}$ be as in Definition 6 . Let $\mathbf{x} \in\{0,1, *\}^{m}$ be defined by

$$
x_{i}= \begin{cases}\text { forced value of } u_{i} & \text { if } u_{i} \text { is forced in } t \text { steps } \\ * & \text { otherwise. }\end{cases}
$$

Since $v$ is not forced in $t+1$ steps, there is some $j$ such that $x_{j}=*$ and $\mathbf{x}^{\prime} \in\{0,1\}^{m}$ such that $x_{i}=x_{i}^{\prime}$ whenever $x_{i} \neq *$ and $f_{v}\left(\mathbf{x}^{\prime}\right) \neq f_{v}\left(\mathbf{x}^{\prime j}\right)$. Since $N_{-}^{t+1}(v)$ induces a tree, by the induction hypothesis, $A_{-}^{t}\left(u_{j}\right) \neq \emptyset$. Then there are states $\mathbf{y}$ and $\mathbf{y}^{\prime}$ such that

$$
\begin{aligned}
& B_{u_{i}}^{t}(\mathbf{y})=B_{u_{i}}^{t}\left(\mathbf{y}^{\prime}\right)=x_{i}^{\prime} \text { for } i \neq j, \text { and } \\
& B_{u_{j}}^{t}(\mathbf{y}) \neq B_{u_{j}}^{t}\left(\mathbf{y}^{\prime}\right) .
\end{aligned}
$$

But then $B_{v}^{t+1}(\mathbf{y}) \neq B_{v}^{t+1}\left(\mathbf{y}^{\prime}\right)$, and $A_{-}^{t+1}(v) \neq \emptyset$. 
We now use another branching process to model the propagation of gates that affect a given gate. The theorems about forced gates are similar to those about ineffective gates, but the proofs are simpler. Therefore we will only sketch them. This time we define the branching process by:

$$
q_{k}=\sum_{i: m_{i} \geq k} p_{i} \sum_{\substack{x \in\{0,1\}^{m_{i}} \\ \gamma\left(\phi_{i}, \mathbf{x}\right)=k}} a^{v(\mathbf{x})}(1-a)^{m_{i}-v(a)}
$$

for $k=0,1, \ldots$ Then $\mu=\lambda$ and by our conditions that $\sum_{i=1}^{\infty} p_{i} m_{i}^{2}<\infty$, $\sigma^{2}<\infty$. Again,

$$
\begin{aligned}
\mathbf{E}\left(Z_{t}\right) & =\lambda^{t}, \text { and } \\
\operatorname{var}\left(Z_{t}\right) & = \begin{cases}\frac{\sigma^{2} \lambda^{t}\left(\lambda^{t}-1\right)}{\lambda^{2}-\lambda} & \text { if } \mu \neq 1, \\
t \sigma^{2} & \text { if } \mu=1 .\end{cases}
\end{aligned}
$$

Definition 7. Let $T$ be a labelled tree of height $t, B=\langle V, E, \mathbf{f}\rangle$ be a Boolean network, and $\mathbf{x} \in\{0,1\}^{n}$ be its state. For $v \in\{1, \ldots, n\}$, we put $T \Longrightarrow v$ if

$A_{-}^{t}(v, \mathbf{x})$ induces a tree in $\langle V, E\rangle$, and

there is an isomorphism from $T$ onto $\left\langle A_{-}^{t}(v, \mathbf{x}), E\right\rangle$.

Lemma 6. If $|T| \leq n^{\beta}$ and the height of $T$ is $t \leq \alpha \log n$, then for all $\mathbf{x} \in$ $\{0,1\}^{n}, \operatorname{pr}(T \Longrightarrow v)=\operatorname{bpr}(P \uparrow t \cong T)(1+o(1))$.

Theorem 5. Let $\chi$ be a property of branching processes that depends only on the first $\alpha \log n$ generations. Then for all $\mathbf{x} \in\{0,1\}^{n}$

$$
\operatorname{pr}\left(\left\langle A_{-}^{t}(v, \mathbf{x}), E\right\rangle \models \chi\right)=\operatorname{bpr}(P \models \chi)+o(1) .
$$

Theorem 6. There is a constant $r$ such that for all $\mathbf{x} \in\{0,1\}^{n}$

$$
\lim _{n \rightarrow \infty} \operatorname{pr}(v \text { is forced in } \alpha \log n \text { steps })=r .
$$

When $\lambda \leq 1, r=1$, and when $\lambda>1, r<1$.

Corollary 4. The expected number of gates forced in $\alpha \log n$ steps in a random Boolean network is asymptotic to rn.

Corollary 5. The number of gates forced in $\alpha \log n$ steps in almost all Boolean networks is asymptotic to rn.

That is, there is a function $\varepsilon(n)$ such that $\varepsilon(n) \rightarrow 0$ and, letting the random variable $X_{n}$ be the number of gates forced in $\alpha \log n$ steps in a random Boolean network with $n$ gates,

$$
\lim _{n \rightarrow \infty} \operatorname{pr}\left(\left|X_{n}-r n\right| \leq n \varepsilon(n)\right)=1 .
$$

When $\lambda>1$, it is also true that most of the gates that are not forced in $\alpha \log n$ steps are affected by many other gates.

Corollary 6. Let $\lambda>1$. For almost all random Boolean networks, if gate $v$ is not forced in $\alpha \log n$ steps, then there is a positive $W$ such that for $t \leq \alpha \log n$, the number of gates that affect $v$ at time $t$ is asymptotic to $W \lambda^{t}$. 


\section{Networks of 2-Input Gates}

Since Kauffman's nets are special cases of our networks, a natural question is whether our analysis agrees with the conclusions of his experiments. We will apply our results to the two classes of 2-input random Boolean networks that Kauffman studied. In one case, we have agreement: the networks are stable with high probability. In the other case, our theorems do not contradict the experimental results, but they provide evidence that these networks are less stable than the first kind. Further, the conjecture that random Boolean networks with a high proportion of canalyzing gates are stable is not supported by our analysis. To explain these results, we use the notion of canalyzing to classify the 2-argument Boolean functions. A Boolean function $f\left(x_{1}, \ldots, x_{m}\right)$ is canalyzing if it is forced by some $\mathbf{x} \in\{0,1, *\}^{m}$ where $x_{i} \neq *$ for exactly one $i \in\{1, \ldots, m\}$. We have three categories of 2 -argument Boolean functions:

I. The two constant functions:

$$
f\left(x_{1}, x_{2}\right)=0 \quad \text { and } \quad f\left(x_{1}, x_{2}\right)=1
$$

II. The twelve nonconstant canalyzing functions, consisting of

A. The four functions that depend on one argument:

$$
\begin{aligned}
& f\left(x_{1}, x_{2}\right)=x_{1} \quad \text { and } \quad f\left(x_{1}, x_{2}\right)=\neg x_{1} \\
& f\left(x_{1}, x_{2}\right)=x_{2} \quad \text { and } \quad f\left(x_{1}, x_{2}\right)=\neg x_{2}
\end{aligned}
$$

B. The eight canalyzing functions that depend on both arguments:

$$
\begin{aligned}
& x_{1} \vee x_{2} \quad \text { and } \quad \neg x_{1} \wedge \neg x_{2} \\
& \neg x_{1} \vee x_{2} \quad \text { and } \quad x_{1} \wedge \neg x_{2} \\
& x_{1} \vee \neg x_{2} \quad \text { and } \quad \neg x_{1} \wedge x_{2} \\
& \neg x_{1} \vee \neg x_{2} \quad \text { and } \quad x_{1} \wedge x_{2}
\end{aligned}
$$

III. The two noncanalyzing functions exclusive or and equivalence:

$$
x_{1} \oplus x_{2} \quad \text { and } \quad x_{1} \equiv x_{2}
$$

Since each function is paired with its negation, Equation 1 is satisfied with $a=1 / 2$. Let $p_{\mathrm{I}}, p_{\mathrm{II}}$, and $p_{\mathrm{III}}$ be the respective sums of the probabilities of the functions of type I, II, and III, i.e., $p_{\text {I }}$ is the probability that a gate is assigned a function of type $\mathrm{I}$, and so on. We can now express the $\lambda$ parameter of Section 4 (see Equation (3)) in terms of $p_{\mathrm{I}}, p_{\mathrm{II}}$, and $p_{\mathrm{III}}$. Since $a=1 / 2$, $a^{v(\mathbf{x})}(1-a)^{2-v(\mathbf{x})}=1 / 4$ for every $\mathbf{x} \in\{0,1\}^{2}$. Clearly, if $\phi_{i}$ is of type I,

$$
\gamma\left(\phi_{i}, \mathbf{x}\right)=0 \text { for every } \mathbf{x} \in\{0,1\}^{2} .
$$

If $\phi_{i}$ is of type II.A., say $\phi_{i}\left(x_{1}, x_{2}\right)=x_{1}$, then

$$
\gamma\left(\phi_{i}, \mathbf{x}\right)=1 \text { for every } \mathbf{x} \in\{0,1\}^{2} .
$$


If $\phi_{i}$ is of type II.B., say $\phi_{i}\left(x_{1}, x_{2}\right)=x_{1} \vee x_{2}$, then

$$
\sum_{\mathbf{x} \in\{0,1\}^{2}} \gamma\left(\phi_{i}, \mathbf{x}\right)=4
$$

Altogether, the type I and II functions contribute $p_{\text {II }}$ to $\lambda$. Lastly, it is easily seen that if $\phi_{i}$ is a type III function, then

$$
\gamma\left(\phi_{i}, \mathbf{x}\right)=2 \text { for every } \mathbf{x} \in\{0,1\}^{2},
$$

and therefore the type III functions contribute $2 p_{\text {III }}$ to $\lambda$, giving

$$
\lambda=p_{\mathrm{II}}+2 p_{\mathrm{III}} .
$$

By Corollaries 2 and 5, for almost all Boolean networks, almost all gates are $\alpha \log n$-ineffective if and only if $\lambda \leq 1$, and for almost all Boolean networks, almost all gates are forced in $\alpha \log n$ steps if and only if $\lambda \leq 1$. Since $\lambda=$ $p_{\mathrm{II}}+2 p_{\mathrm{III}}$ and $p_{\mathrm{I}}+p_{\mathrm{II}}+p_{\mathrm{III}}=1, \lambda \leq 1$ is equivalent to $p_{\mathrm{I}} \geq p_{\mathrm{III}}$. Therefore both types of ordered behavior hold if and only if $p_{\text {I }} \geq p_{\text {III }}{ }^{1}$

Kauffman performed extensive simulations on two classes of random networks constructed from 2-argument Boolean functions. In the first class, all 16 of these functions were equally likely to be assigned to a gate. In the second, no constant functions were used, and the remaining 14 functions were equally likely. In the first case, $p_{\mathrm{I}}=p_{\mathrm{III}}=1 / 8$. Therefore in this case, almost all gates are $\alpha \log n$-ineffective and forced in $\alpha \log n$ steps. But in the second case, $p_{\mathrm{I}}=0$ and $p_{\text {III }}=1 / 7$, so $p_{\text {I }}<p_{\text {III }}$. Thus in this case, a nontrivial fraction of the gates are $\alpha \log n$-effective and not forced in $\alpha \log n$ steps. This does not directly contradict the experimental conclusions because it is possible that, even though the effect of a perturbation persists for $\alpha \log n$ steps, it could die out after that. And even if it persisted indefinitely, it may not change the limit cycle that the network enters. Similarly, a gate may not be forced, but it could still freeze.

\section{Future Work and Open Problems}

In [16], it was shown that, for random Boolean networks with 2-input gates, limit cycles are bounded in size with high probability when $p_{\text {I }}>p_{\text {III }}$. But in [17], it was shown that limit cycles are large at the threshold $p_{\text {I }}=p_{\text {III }}$. Specifically, the average size of the limit cycle is greater than $n^{a}$ for any $a$. This disagrees with Kauffman's claim that the average limit cycle size is on the order of $\sqrt{n}$.

These results generalize to the classes of random Boolean networks considered in this paper: when $\lambda<1$, limit cycles are bounded in size with high probability, but when $\lambda=1$, the average size of the limit cycle is superpolynomial in the number of gates. It is reasonable to conjecture that the limit cycle size is monotonic in $\lambda$, and that limit cycles would be very large when

\footnotetext{
${ }^{1}$ Article [17] contains proofs that $p_{\mathrm{I}} \geq p_{\text {III }}$ implies these kinds of ordered behavior; it was conjectured in [17] that they fail when $p_{\text {I }}<p_{\text {III }}$.
} 
$\lambda>1$. This would also agree with the experimental evidence. However, we do not know of a proof.

Other conjectures about the long-term behavior of nets with $\lambda>1$ are unresolved. Based on the results of this article, one would expect that the other forms of instability would be prevalent: sensitivity to initial conditions and many unfrozen gates.

¿From what we have shown, $\lambda=1$ appears to be the point at which complex behavior begins. This idea could be investigated further by considering Boolean networks with inputs and outputs. These are an easy generalization of the networks studied here. Such networks can compute functions of their inputs, and we conjecture that when $\lambda<1$, these functions are very simple with high probability, but when $\lambda=1$, the functions can be artibrarily complex in the senses of computational complexity.

\section{References}

[1] P. Bak, How Nature Works: The Science of Self-organized Criticality, Oxford University Press (1997).

[2] B. Bollobás, Random Graphs, Academic Press (1985).

[3] J. M. Bower and H. Bolouri, Computational Modeling of Genetic and Biochemical Networks, The MIT Press, Cambridge, MA (2001).

[4] J. E. Cohen and T. Łuczak, Stability of vertices in random Boolean cellular automata, Random Structures and Algorithms 2 (1991), 327-334.

[5] B. Derrida and Y. Pomeau, Random networks of automata: a simple annealed approximation, Europhys. Lett. 1 (1986), 45-49.

[6] P. Erdős and A. Rényi, On random graphs, Pub. Math. 6 (1959), 290-297.

[7] P. Erdős and A. Rényi, On the evolution of random graphs, Magyar Tud. Akad. Mat. Kutato Int. Kozl. 5 (1960), 17-61.

[8] E. Gilbert, Random plane networks, J. SIAM 9 (1961), 533-543.

[9] B. Harris, Probability distributions related to random mappings, Ann. Math. Stat. 31 (1960), 1045-1062.

[10] T. E. Harris, The Theory of Branching Processes, Dover Publications, Inc., New York (1989).

[11] F. Jacob and J. Monod, Genetic repression, allosteric inhibition ad cellular differentiation, in Cytodifferentiation and Macromolecular Synthesis, M. Locke, ed., Academic Press, New York (1963), 30-64.

[12] S. Jaffe, Kauffman networks: Cycle structure of random clocked Boolean networks, Ph. D. diss., New York University (1988). 
[13] S. A. Kauffman, Metabolic stability and epigenesis in randomly connected nets, J. Theoret. Biol. 22 (1969), 437-467.

[14] S. A. Kauffman, The Origins of Order: Self-Organization and Selection in Evolution, Oxford University Press, New York, (1993).

[15] C. G. Langton, Computation at the edge of chaos: phase transitions and emergent computation, Physica D 42 (1990), 12-37.

[16] J. F. Lynch, A criterion for stability in random Boolean cellular automata, Ulam Quart. 2 (1993), 32-44.

[17] J. F. Lynch, On the threshold of chaos in random Boolean cellular automata, Random Structures and Algorithms 6 (1995), 239-260.

[18] J. F. Lynch, Critical points for random Boolean networks, Physica D 172 (2002), 49-64.

[19] M. Molloy and B. Reed, A critical point for random graphs with a given degree sequence, Random Structures and Algorithms 6 (1995), 161-179.

[20] M. Molloy and B. Reed, The size of the giant component of a random graph with a given degree sequence, Combinatorics, Probabilitty and Computing 7 (1998), 295-305.

[21] M. E. J. Newman, S. H. Strogatz, and D. J. Watts, Random graphs with arbitrary degree distributions and their applications, Phys. Rev. E 64 (2001), 026118 .

[22] N. H. Packard, Adaptation toward the edge of chaos, in Dynamic Patterns in Complex Systems, J. A. S. Kelso, A. J. Mandell, and M. F. Shlesinger, eds., World Scientific, Singapore (1988), 293-301.

[23] D. J. Watts and S. H. Strogatz, Collective dynamics of "small world" networks, Nature 393 (1998), 440-442.

[24] D. J. Watts, Small Worlds: The Dynamics of Networks Between Order and Randomness, Princeton University Press, Princeton, NJ (1999).

[25] S. Wolfram, A New Kind of Science, Wolfram Media Inc., Champaign, IL (2002). 